\title{
The Hulk: Design and Development of a Weather-proof Vehicle for Long-term Autonomy in Outdoor Environments
}

\author{
Stephen Kyberd, Jonathan Attias, Peter Get, Paul Murcutt, Chris Prahacs, Matthew \\ Towlson, Simon Venn, Andreia Vasconcelos, Matthew Gadd, Daniele De Martini, \\ Paul Newman
}

\begin{abstract}
In recent years the range of robotics platforms available for research and development has increased dramatically. Despite this there are areas and applications which are not currently well served by the existing available platforms. Many of them are designed for indoor use; the range of outdoor and off-road robotics platforms is less diverse and few address the issue of deployment in hazardous weather conditions and integrate a suitable sensor suite. In addition almost all of the commercially available Unmanned Ground Vehicles (UGVs) are unsuitable for deployment on delicate surfaces. Given the widespread use of manicured grass within the built environment and agriculture across the western world, this severely limits where they can be deployed and the tasks that can be accomplished. This paper introduces the design principles of a suitable autonomous vehicle. Hulk is built from a commercial zero-turn mower modified for fly-by-wire operation and equipped with a full sensor suite and computing payload. To enable remote, long-term autonomy in diverse environments, several layers of redundant safety systems were designed and installed and the entire assembly made weatherproof.
\end{abstract}

\section{Introduction}

There is a large and growing need for autonomous robots to perform a wide range of practical tasks in the near future. These span a wide range of industries, from autonomous cars and transportation to robots for hazardous environments such as oil fields [8]. A plethora of platforms are available for research and development in many of these areas and some are available for commercial deployment. These

Oxford Robotics Institute, 23 Banbury Road, Oxford

e-mail: $\quad$ skyberd, jattias, peterget, pmurcutt, cprahacs, matthew, svenn, andreia, mattgadd, daniele, pnewman\}@robots.ox.ac.uk 
include mobile indoor robots [18], off road robots such as those from Clearpath Robotics 1 and even explosion-proof robots [10].

Robots designed to be deployed into an outdoor environment span a wide range of sizes and capabilities. For example from Clearpath they range from the Jacka ${ }^{2}$ to the Moose ${ }^{3}$ Due to the simplicity of control almost all outdoor robots currently available use skid steering, but this means that on damp ground, even moderately tight turns are liable to damage the surface. While acceptable for industrial applications or rough terrain use, this is entirely unacceptable for many other applications, such as agriculture or operations on manicured grassed areas. This limits the ability to deploy robots into a wide range of potential applications; including security patrols, transport, and lawn or pitch maintenance.

This paper describes the engineering design principles used to realise an Unmanned Ground Vehicle (UGV) suitable for operation over extended time-scales without expert supervision. We build on our experience in past projects - including the very early Segway - Lisa [17], the offroading Bowler Wildcat [16], the urban Nissan Leaf RobotCar [9], and light transport LUTZ Pathfinder pod [12] - and detail the holistic approach taken in order to meet a set of requirements dictated by a third-party group of clients which require the robot to operate in a challenging, unstructured, grassy environment.

This work is structured as follows. Section 2 delineates the system requirements for the platform, while Section 3 describe the process that led to the choice of the base vehicle for the robot. Section 4 and Section 5 describe the two main groups of components installed on the platform. The mechanical modifications needed are covered in Section 6. Finally, the Human-Robot Interaction (HRI) system installed on the platform is described in Section 7 .

\section{Design Guidelines}

When setting out to create the robotic vehicle, a set of extensive design requirements were created that helped to guide the process. We refer to the retrofitted platform that we designed to fulfill these requirements as the Hulk. At a high level, these requirements included:

Long-term autonomous operation: The key requirement is that the vehicle should be able to operate for long periods - weeks or months - reliably and unattended, except for being put on charge. It must perform tasks like inspection and transport in diverse and unstructured environments and weather conditions. This influenced the choice of the base vehicle and waterproofing requirements(c.f. Section 6; ; as well as the components installed, like the Main Vehicle Computer (MVC) and sensor payload (c.f. Section 6).

\footnotetext{
${ }^{1}$ Clearpath Robotics Inc.: https://www.clearpathrobotics .com

2 Jackal platform: https://bit.1y/2I3JzRP

${ }^{3}$ Moose platform: https://bit.ly/2uIrgc7
} 
Terrain capabilities: To enable the widest possible scope for deployment the vehicle should have little or no impact on the surface it is driving on. This heavily influenced the choice of base vehicle (c.f. Section 3).

Human safety: The resulting platform is a large and relatively quick vehicle: the safety of humans and objects around it is of primary concern. A hardware and software safety stack is designed (c.f. Section 5) and bystander interaction considered (c.f. Section 7).

Simplicity of use: Non-technical users should be able to operate and work alongside the Hulk with minimal prior knowledge; a HRI system has been added to facilitate this (c.f. Section 7).

\section{Base Vehicle}

A key requirement is that the robot is able to operate for weeks or months reliably. A Commercial Off-The-Shelf (COTS) vehicle, with custom modifications, was therefore preferred over a completely custom design. This ensured a sturdy and reliable foundation platform which has already passed the tests necessary for commercialisation. This choice had the benefit of speeding up development.

The requirement for minimal terrain impact focused the search for a base vehicle into lawn mowers, which are designed for outdoor operations on delicate terrains. The so called zero-turn mowers were of primary interest because of the simplicity of adapting the control interface. These mowers are characterised by two large drive wheels at the rear and two castor wheels at the front. They use differential control of the rear wheels to maneuver without the use of a steering rack; which we would otherwise need to mechanically actuate.

Commercial products were favoured over domestic products given their improved durability and longer run times. The requirement for electric power for the Automonous Control System (ACS) payload demanded an electric powertrain vehicle; this has the added benefit of zero exhaust fumes and low running noise enabling humans to work in close proximity without discomfort.

The platform chosen is an SK-48 Stalker built by Mean Green Mowers 4 This fulfilled all requirements of size, battery life, and smooth operation on grass. This is shown in Figure 1.

\section{Sensor and Computer Payload}

The Hulk is equipped with a range of different sensors of heterogeneous modalities. This provides redundancy and resilience in its operation with each complementing the next. Their general layout is shown in Figure 2.

\footnotetext{
${ }^{4}$ Mean Green Mowers : SK-48 Stalker: https://meangreenproducts.com/sk48-stalker/
} 


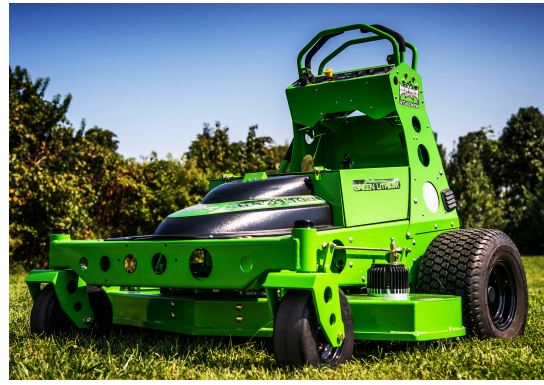

(a)

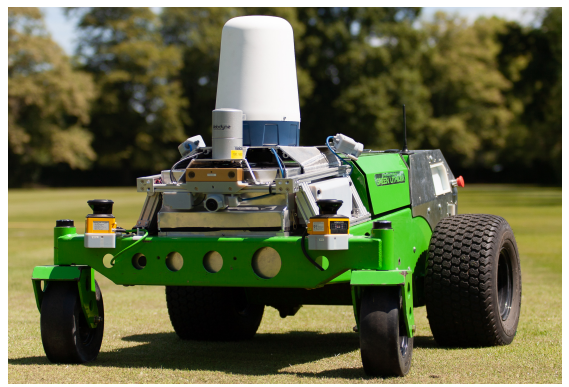

(b)

Fig. 1 Photos of the platform before (a) and after (b) modifications. The modifications are clearly visible with the sensors at the front and the power and control circuitry compartment at the rear

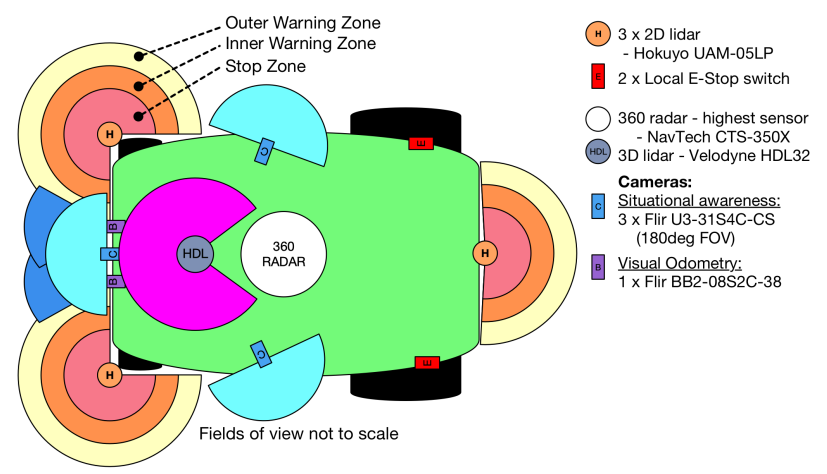

Fig. 2 The fields of view and arrangement of the primary and SCS sensors

The main requirement of the platform, as described in Section 2, is to be capable of autonomous operation in any weather. For this reason, the main sensor for perception is a CTS350-X Navtech Frequency-Modulated Continuous-Wave (FMCW) scanning radat 5 , which is mounted on top of the platform with an axis of rotation perpendicular to the driving surface. It is characterised by an operating frequency of $76 \mathrm{GHz}$ to $77 \mathrm{GHz}$, yielding up to 2000 range readings with a resolution of $0.175 \mathrm{~m}$, each constituting one of 400 to 800 azimuth readings - each $2^{\circ}$ wide - and a scan rotation rate of $4 \mathrm{~Hz}$ to $8 \mathrm{~Hz}$. Compared to other sensors, like cameras and lidars, the radar suffers far fewer ill effects from deleterious weather conditions such as rain, fog, or snow. This increases the Hulk's resilience and reliability in all conditions. The radar can be used for obstacle detection, odometry and localisation [6, 4]

The platform is also equipped with a number of cameras and a lidar to perceive the world. Mounted on the front of the platform facing towards the direction of motion is a Point Grey Bumblebee2 stereo camerd 6 The camera is characterised by

${ }^{5}$ CTS350-X Navtech scanning radar: https://bit.ly/2Ur5yI0

${ }^{6}$ Bumblebee2 camera: https://bit.1y/2KmTOh5 
$1032 \times 776 \times 3$ resolution, $20 \mathrm{~Hz}, 1 / 3^{\prime \prime}$ Sony ICX204 CCD, global shutter, $3.8 \mathrm{~mm}$ lens, $65^{\circ} \mathrm{HFoV}, 12 \mathrm{~cm}$ baseline. Its primary tasks are Visual Odometry (VO) [11] and forward obstacle and terrain assessment [5].

Three Point Grey monocular camera: 7 with fisheye lenses are mounted on the platform. These cameras are characterised by $2048 \times 1536 \times 3$ resolution, $55 \mathrm{~Hz}$, $1 / 1.8^{\prime \prime}$ Sony IMX265 CCD, global shutter. The main role of the cameras is used for obstacle detection and situational awareness for when the Hulk is being teleoperated.

Further to these sensors, the platform is equipped with a Velodyne HDL-32E Lidar ${ }^{8}$ which presents a $80 \mathrm{~m}$ to $100 \mathrm{~m}$ range, $360^{\circ}$ horizontal Field of View (FoV), and $40^{\circ}$ vertical $\mathrm{FoV}$. This sensor is primarily used for obstacle detection.

In addition to the aforementioned sensors, the platform is equipped with three safety sensors, which will be covered in more detail in Section 5.1. These are Hokuyo UAM-05LP-T301 laser scanners 9 with a FoV of $270^{\circ}$.

Finally, we include in the sensor payload a 3DM-GX4-45 Microstrain inertial senso ${ }^{10}$ with drift gyros. This allows GPS data to be captured and GPS waypoint planning to be used if desired.

Due to the experimental nature of the system and its likely deployment to sites with low connectivity (even potentially when connected to a base station), the decision was made to support as much compute power on board the Hulk as the mechanical platform would permit. It is equipped with a $2.4 \mathrm{GHz} 10$-core Xeon CPU and Geforce Titan X Graphics Processing Unit (GPU) These permit both online and offline Deep Learning algorithms to be implemented on-board [13, 14]

\section{Safety Systems}

In order to provide sufficient confidence in the safety systems on the platform, the design and implementation was carried out in accordance with the European Machinery directive and associated standards:

- BS EN ISO 12100:2010 [1] - Safety of machinery. General principles for design. Risk assessment and risk reduction

- BS EN ISO 13850:2015 [3] - Safety of machinery. Emergency stop function. Principles for design

- BS EN ISO 13849-1:2015 [2] - Safety of machinery. Safety-related parts of control systems General principles for design

The use of a CE marked COTS baseline made this process simpler as only additional sensors and housings required rating. These standards led us through a risk assessment process to determine the required reliability of the system.

${ }^{7}$ Chameleon cameras: https://bit.1y/2D2Zrjt

${ }^{8}$ Velodine HDL-32E: https://velodynelidar.com/hdl-32e.html

${ }^{9}$ Hokuyo UAM-05LP-T301: https://bit.ly/2I8C1gI

10 3DM-GX4-45 Microstrain inertial sensor: https://bit.ly/2Iouh9M 
The Performance Level (PL) standard defines a methodology for assessing the risk to individuals using or in close proximity to the equipment. This then defines a range of five performance levels that the control system must be built to, from the lowest rating of PL-a through to PL-e. The standard asks three fundamental questions about the risk that the device poses to humans: severity of injury - for Hulk serious, since it is a large and relatively quick vehicle; the frequency of exposure for Hulk seldom, it is designed to work mostly alone; the ability to avoid injury - for Hulk avoidable as it will be operating outside in large open spaces.

This assessment specifies a PL-c rating for the Hulk which requires an average probability of a dangerous failure per hour to be less than $3 \times 10^{-6}$. This informs our design process as will be described in the following sections in which we describe the hardware and software stacks as well as the choice of components for either.

\subsection{Hardware Safety Curtain System}

To meet the required PL-c rating we have implemented a Hardware Safety-Curtain System (HW-SCS) on a set of bespoke Printed Circuit Boards (PCBs) It integrates two main safety functions: obstacle detection and ranging (feeding into commands which slow or stop the vehicle), along with an Emergency Stop (E-Stop) function which can be commanded remotely and locally. To achieve the safety rating, each of the three signal sources are PL-c or PL-d rated COTS components or systems:

- Three 2D, 270 degree FoV lidars, designed for use on forklift trucks and other industrial equipment. These have an essentially uninterrupted $\mathrm{FoV}$ around the robot and provide a series of digital I/O lines to communicate discrete signals and commands.

- E-Stop switche ${ }^{11}$ mounted on each side of the chassis. These feature two independent switch blocks for redundancy.

- A wireless E-Stop system (Tyro Indus $1 \mathrm{~S} / \mathrm{Gemini}$

To achieve the required PL-c rating for safety-stop related functions a Category 3 [2] architecture is used. This requires two independent channels from signal to actuator. Each of the three sources provide pairs of independent outputs which are connected to parallel circuits within the HW-SCS system. To meet the monitoring requirement, all control signals are routed through double pole relays. The relays' second channels are used for diagnostic signals which are routed back to the ACS and/or a user interface. Numerous state and health monitoring signals are provided.

To make use of the mower's existing safety features, the HW-SCS system is designed to interface with the Original Equipment Manufacturer (OEM) dead man signal in a fail-safe manner. Any break in the signals from the switches or sensors results in a break in the dead man signal, the mower's control system then initiates a Category 2 Stop [3], immediately bringing the vehicle to a controlled stop and

11 E-Stop switches: https://www.schneider-electric.com/en/search/XB4BS8444

12 Tyro Indus $1 \mathrm{~S} /$ Gemini: https://bit.ly/2Z1mC79 
applying the motors' brakes, leaving the system powered. Speed signals are ignored by the mower controllers.

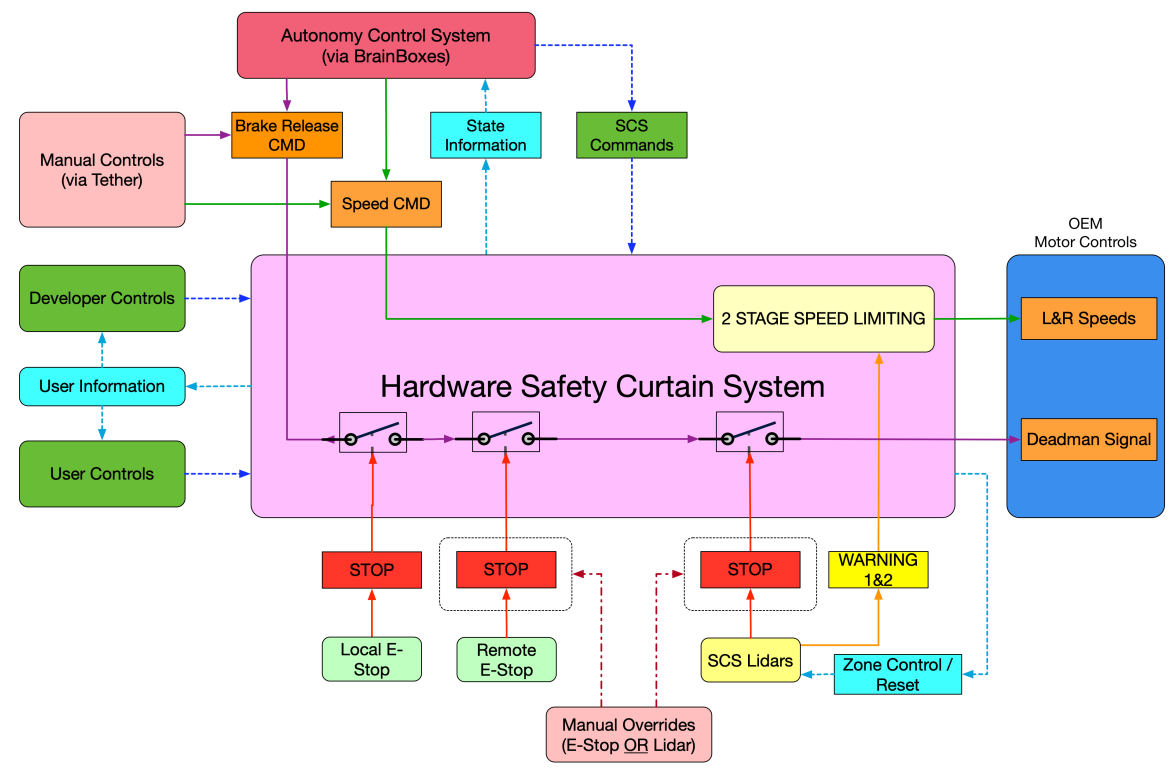

Fig. 3 Simplified block diagram for the HW-SCS

In keeping with the safety standard, when a safety-stop is triggered a separate reset command is required to re-enable control of the vehicle. This prevents the vehicle from restarting the moment the cause of the stop is cleared.

In addition to the safety-critical stop signal, the lidars provide two signals to indicate the presence of obstacles within two warning ranges, these are set outside the safety range. Multiple sets of ranges (zones) can be pre-programmed into each lidar, selected via discrete digital inputs. The HW-SCS makes use of these warning signals to cap the speed command voltages sent to the Hulk in two stages: affording the ACS and bystanders more time to react and avoid obstacles.

The HW-SCS has been designed to accommodate a range of planned use cases including attaching a trailer, vehicle transport, and emergency recovery. The latter is supported through a tether to connect the OEM controls for manual operation of the mower. For vehicle transport, manual operation, or operation in tight spaces; the safety-stop functions of either the remote E-Stop or the lidars can be temporarily overridden via a keyed switch. The speed limiting function is maintained to assist the operator. A specially modified trailer can be attached through a harness which augments the HW-SCS via lidar(s) on the trailer. When the trailer harness and/or the tether are connected, the SCS automatically switches the lidars to appropriate zone 
sizes to allow for the presence of a trailer and/or user, as well as navigation in tight spaces.

\subsection{Software Safety Curtain}

In addition to this hardware level of guaranteed safety we layer on a Software Safety-Curtain System (SW-SCS), The primary purpose is to gently slow the Hulk down when approaching obstacles so that when the HW-SCS is triggered there is no abrupt and sudden change of speed. This behaviour would be alarming for people near the robot and reduces the acceptance of the robot in their workspace. The software therefore sets larger monitoring areas than is done in hardware.

The SW-SCS monitors the range measurements from the same safety lasers used in the hardware safety curtain. When it detects an obstacle it limits the maximum speed command that can be issued. This facilitates a far smoother profile than can be simply and reliably achieved through a hardware-based system.

\section{Vehicle Modification}

The base vehicle is divided into three main compartments: at the rear sit the motors and associated drive circuity, in the middle lie the batteries and at the fore there is a leaf blower. This leaf blower was removed to provide space for the computer and sensor payload. The manual controls and operator platform at the rear were removed and a new compartment was created for additional power and control circuitry. The battery bay was left untouched and unobstructed for ease of access to the batteries.

\subsection{Sensor and Compute Bay}

The front compartment houses the greater portion of the ACS, containing the MVC as well as all the primary sensors and some of the power system. The primary sensors are rigidly attached to a chassis which sits over the MVC compartment. This sensor chassis is detachable from the platform chassis, to give easy access to the MVC. whilst maintaining the relative positions of the primary sensors. This defines the outer volume of the ACS compartment and was designed to prioritise the sensor FoVs

The Computer-Aided Design (CAD) model of the sensor chassis can be seen in Figure 4(a), while the sensor suite is listed and discussed in detail in Section 4. FoVs

There were a number of requirements for the sensor rig based on the required

- The monocular cameras had to cover a full $360^{\circ}$. 
- The radar FoV had to be entirely unobstructed.

- The Velodyne FoV should be maximised, and it should see the ground as close as possible to the vehicle.

- The GPS antenna should have an unobstructed view of the sky.

- The BumbleBee 2 needs to be mounted facing forward with a clear view of the ground.

The sensor positions were initially modelled in CAD with their FoVs visualised for fine tuning of their positions. A temporary rig was constructed with the sensors placed as in the CAD model. Field testing was then carried out and data collected for verification of these positions. Adjustments were quick and easy and could be performed in the field. During the design of the $\mathrm{MVC}$ enclosure some sensors did have to be marginally moved from these positions but the changes were approved using the rig and further data collections.

The sensor chassis was built out of Series 5 Metallin and welded plate metal to keep it lightweight and rigid. Four countersunk screws attach it to the platform chassis to allow rapid removal. Removal is kept simple through the use of a single power and data umbilical cord to link it to the ACS compartment. This allows the sensor chassis to be placed next to the platform chassis when removed.

To enable hardware troubleshooting without removing the sensor chassis, two break-out boxes were designed. These are situated on either side of the sensor chassis and provide access to USB and Ethernet ports from sensors and the MVC In the event of a malfunctioning sensor these can be used to diagnose whether the fault is likely to be a hardware fault with the sensor itself or a hardware or software fault with the MVC. Only if the MVC is found to be at fault does the sensor rig need removing.

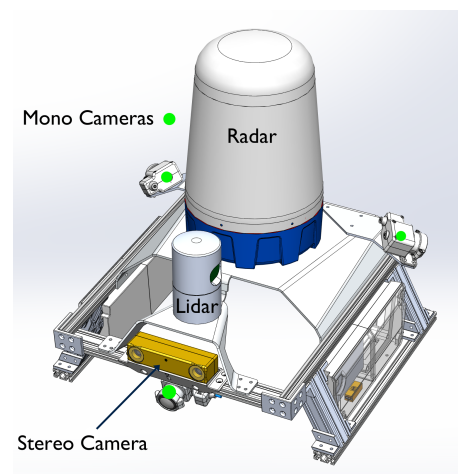

(a)

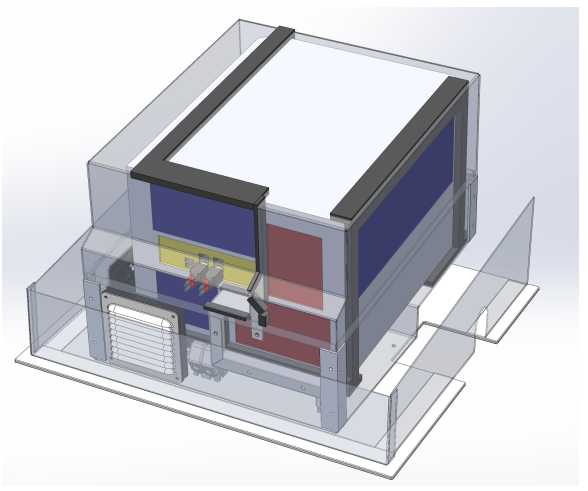

(b)

Fig. 4 CAD models of the sensor chassis (a) and MVC compartment (b). This shows the independent sensor rig formed of Mettalin in (a) and the airflow control in the MVC compartment with inlets in blue and outlets in red. 
Between the sensor chassis and the cutting deck, a compartment that can fit and cool the computer and GPU was required. The airflow in the compartment is carefully controlled to ensure the correct flow through the multiple air inlets and outlets of the MVC See Figure 4(b) The movement of air is controlled using baffles formed from sheet metal and closed cell foam. This guides the air through the MVC over the power transformers and out.

The built-in cooling system of the MVC blows air out so additional fans were added at the inlets to suck cold air in. The required fan specifications was calculated to limit the maximum temperature to $45^{\circ} \mathrm{C}$ in an ambient temperature of $18^{\circ} \mathrm{C}$. This requires an airflow of $25 \times 10^{-2} \mathrm{~m}^{3} \mathrm{~s}^{-1}$. Redundancy is provided through dual fans, each capable of delivering this airflow.

Due to the required airflow, space limitations, and ease of access to the enclosure the maximum IP rating attained was IP54. It is intended that a shroud that will sit over the sensor chassis will increase the IP rating but this is yet to be designed. The combination of these three main components allow for a rating of IP55.

\subsection{Rear Interface Compartment}

At the rear of the Hulk the redundant manual controls were removed. The rear was slightly lengthened and enclosed to provide a compartment for control circuitry. Two lockable control panels are provided to allow differing levels of access for operators or expert users. A sub frame and hitch were added to allow towing of a trailer. This was situated to work well with standard garden trailers whilst ensuring it does not impinge on the maximum attack angle the Hulk can achieve. This sub frame was created in $\mathrm{CAD}$ and analysed using a Finite Element Analysis (FEA) package. This was iterated to ensure a minimum factor of safety of 3 .

\subsection{Control Interface}

The control interface is considerably simplified through the choice of base vehicle. There is no steering rack or steered wheels to mechanically control; the directional control is provided by the differential speed of the two large rear wheels. As built the speed of these wheels are governed by control sticks that drive two potentiometers. The voltage output of these is the demand signal for each motor controller's torque controller. To convert the Hulk to autonomous operation these manual control lines are intercepted so the ACS can send control signals to the wheels. The OEM quadrature encoders fitted to the wheels are monitored and wheel speeds fed back to the main vehicle computer through an Arduino at $100 \mathrm{~Hz}$. A standard Proportional-Integral-Derivative (PID) controller is then run to provide speed control of the wheels. 
Commercial I/O modules ${ }^{13}$ are used to output the control signal voltages from the ACS. These are configured to run a watchdog which resets the outputs to known safe values if communication with the $\mathrm{MVC}$ is lost. In the case of a power event or software failure that causes the MVC|to hang these reset after $0.2 \mathrm{~s}$ bringing the Hulk to an immediate halt.

\section{Human Robot Interaction}

HRI is a growing area of interest and research as robots move out of the laboratory. Scholtz [15] defines five distinct interaction categories: Supervisor, Operator, Mechanic, Peer, and Bystander. Roles for which special provision has been included are discussed below.

\subsection{Bystander Interaction}

The most important role for the Hulk operation is the Bystander role, which includes whomever the platform shares an environment with. The robot will react to the bystander's actions but the goal or intention of the robot cannot be changed by them. Furthermore the bystander is not expected to have any knowledge of the vehicle, the details of its operation or purpose and may never have seen it before. This means that the bystander actions are completely unpredictable.

We propose three means of seamless interaction with the platform:

- an intuitive and easy to understand display of the Hulk's intentions and knowledge;

- an auditory method of indicating the Hulk's presence and motion;

- the Hulk must demonstrate competence, confidence and purpose in its actions through smooth and deliberate motions.

The first requirement is met through the installation, around the platform, of a ring of LEDs, which indicates that the Hulk has seen a human or obstacle in its SWSCS The software safety curtain is run on the MVC and the location of obstacles sent to an arduino that controls the LEDs. These are lit green unless an obstacle is in the SCS warning zone, when the LEDs that point to the obstacle are lit orange. If the obstacle comes closer to the Hulk and enters the stop zone, the LEDs change to red. This allows the bystander to have confidence when approaching or passing the Hulk that it is aware of their presence and they can carry on safely. If communication with the MVC fails the LEDs will blink red to indicate a fault condition.

The second means of interaction is based on sound. A sounder has been installed on the top of the platform so that the Hulk's approach can be signalled to bystanders,

${ }^{13}$ Brainbox Ethernet I/O module: http://www . brainboxes .com/ethernet-io-module 
including those who are visually impaired. A critical characteristic for the emitted sound is a balance between adequately warning people of the Hulk's approach, whilst not being too irritating for bystanders.

The third and final requirement is met with two different methods. On one hand, the Hulk's acceleration and jerk are limited to maintain smooth velocity rates, with the added benefit of ensuring softer interaction with the terrain. On the other hand, the SW-SCS enforces a gentle slowing of the robot when approaching an obstacle, which again signals the Hulk has seen and is reacting. Combined these ensure the Hulk responds gradually and gracefully to people in its orbit, whilst the HW-SCS can enforce rapid deceleration when required.

\subsection{Supervisor and Operator Interaction}

The Hulk will operate independently and autonomously; nevertheless, operator oversight is required for testing and commissioning. This is facilitated through a software application called the Small Robots Command Centre (SRCC) which is run on a remote computer and connects to the robot wirelessly, allowing them to monitor the platform and send command signals. The MVC is equipped with a wireless card and wireless antenna to enable this (c.f. Section 4 ).

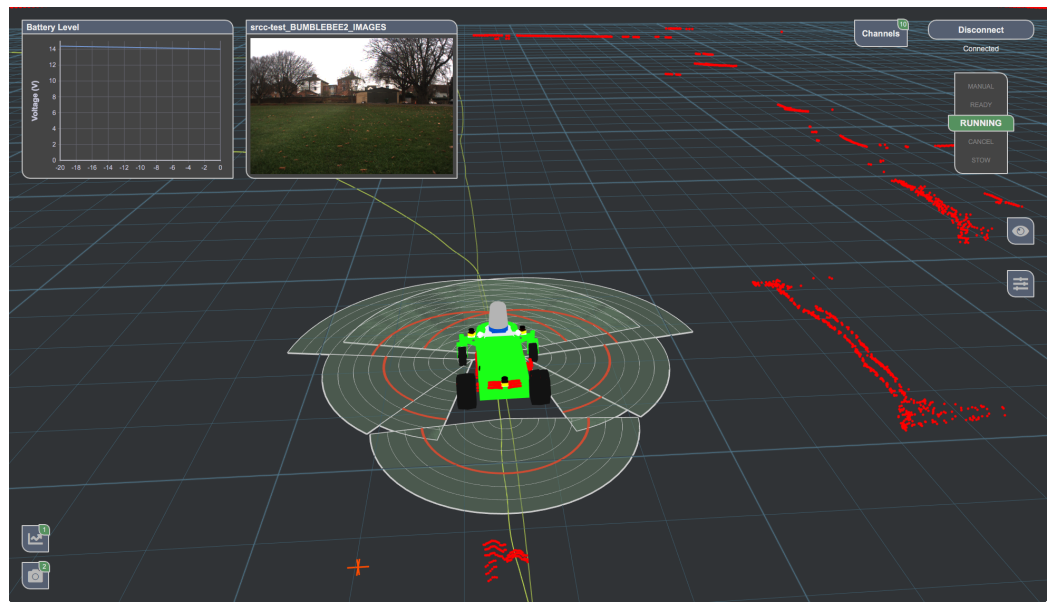

Fig. 5 Screenshot of SRCC remote monitoring software in operation outside at the test site. The safety lasers and SW-SCS state are shown in the 3D workspace and state variables are displayed in overlays. Visualisation elements can be easily turned on and off.

Figure 5 shows a screenshot of the application in operation. The user interface has been designed with touch screens in mind to facilitate the use of tablet computers in the field. The main part of the display is filled with a 3D representation of the robot, its current state, and its immediate environment; including the projected laser 
scans, radar scans, odometry trace and planned route. The SCS state is shown with projected cones marking out the safety zones in each laser, which share the same color scheme as the HRI LEDs that ring the vehicle. The autonomy state is always clearly visible and other internal robot state variables are easily accessed from a pop-up panel.

\subsection{Mechanic Interaction}

Although the Hulk will primarily work autonomously when deployed, daily interactions with mechanics are required when the Hulk needs to be charged and data downloaded for analysis. This is the only manual intervention required in a proposed operational deployment of the Hulk, where at all other points the vehicle will be operating autonomously.

To facilitate these tasks, a dedicated light on the top of the platform is connected directly to the HW-SCS, as mentioned in Section 5.1. This visually indicates that the Hulk is prevented from moving by the HW-SCS allowing the mechanic to safely approach and get into close proximity of the robot.

The same visual approach has been used to guide the required steps for connecting the Hulk to power for charging and downloading collected data. Two blue lights indicate that the power supply has been connected - one for batteries and one for the ACS - a green LED indicates that the Ethernet networking is working properly.

\section{Conclusions and Future Work}

In this paper we described the design and implementation of a robotic platform for long-term autonomous operation in outdoor environments, impervious to adverse weather and lighting conditions. A further requirement was to allow the robot to operate on delicate surfaces, as the ones in agriculture fields or in manicured lawns. Safety and a ease of use were fundamental characteristics of the platform too.

The design started from the choice of a base vehicle, which was chosen to be a zero-turn mower, then modified for fly-by-wire operation and equipped with a full sensor suite and computing payload. To enable remote long-term autonomy in diverse environments, several layers of redundant safety systems were designed and installed and the entire assembly was designed to be weatherproof.

In the immediate future, the computing hardware (MVC and GPU) as described in this paper will be used to support vision and radar algorithms requisite for a mapping and localisation system. These will be integrated into the software stack and tested in a kilometre-scale area defined by an extensive set of lawns and managed by a staff of groundskeepers, with whom Hulk will have to interact with.

We may also design a charging station and procedure to achieve completely autonomous charging (perhaps through the use of inductive charging) - capturing the entire operational cycle. From a perspective as described in [7], lifelong mapping 
and localisation leads to issues around data management and harvesting. These will have to be addressed, as the Hulk is expected to operate continuously, and has a finite storage capability.

\section{References}

1. ISO 12100:2010. International Organization for Standardization: Safety of machinery - General principles for design. Risk assessment and risk reduction. ISO, 2010.

2. ISO 13849-1:2015. International Organization for Standardization: Safety of machinery Safety-related parts of control systems - Part 1: General principles for design. ISO, 2015.

3. ISO 13850:2015. International Organization for Standardization: Safety of machinery Emergency stop function. Principles for design. ISO, 2015.

4. Roberto Aldera, Daniele De Martini, Matthew Gadd, and Paul Newman. Fast Radar Motion Estimation with a Learnt Focus of Attention using Weak Supervision. In IEEE International Conference on Robotics and Automation (ICRA), May 2019.

5. Laszlo-Peter Berczi, Ingmar Posner, and Timothy D Barfoot. Learning to assess terrain from human demonstration using an introspective gaussian-process classifier. In 2015 IEEE International Conference on Robotics and Automation (ICRA), pages 3178-3185. IEEE, 2015.

6. S. Cen and P. Newman. Precise ego-motion estimation with millimeter-wave radar under diverse and challenging conditions. In 2018 IEEE International Conference on Robotics and Automation (ICRA), May 2018.

7. Matthew Gadd and Paul Newman. The Data Market: Policies for Decentralised Visual Localisation. ArXiv e-prints, January 2018.

8. Kris Kydd, Serge Macrez, Pascal Pourcel, et al. Autonomous robot for gas and oil sites. In SPE Offshore Europe Conference and Exhibition. Society of Petroleum Engineers, 2015.

9. Will Maddern, Geoffrey Pascoe, Chris Linegar, and Paul Newman. 1 year, $1000 \mathrm{~km}$ : The oxford robotcar dataset. The International Journal of Robotics Research, 36(1):3-15, 2017.

10. Keiji Nagatani, Daisuke Endo, Atsushi Watanabe, and Eiji Koyanagi. Design and development of explosion-proof tracked vehicle for inspection of offshore oil plant. In Field and Service Robotics, pages 531-544. Springer, 2018.

11. David Nistér, Oleg Naroditsky, and James Bergen. Visual odometry for ground vehicle applications. Journal of Field Robotics, 23(1):3-20, 2006.

12. Alan Peters. Safety of the lutz pathfinder automated vehicle. In Proceedings 22nd ITS World Congress, Bordeaux, France. ERTICO (ITS Europe), 2015.

13. Horia Porav, Tom Bruls, and Paul Newman. I can see clearly now : Image restoration via de-raining. CoRR, abs/1901.00893, 2019.

14. Horia Porav, Will Maddern, and Paul Newman. Adversarial training for adverse conditions: Robust metric localisation using appearance transfer. In 2018 IEEE International Conference on Robotics and Automation (ICRA), pages 1011-1018. IEEE, 2018.

15. Jean C. Scholtz. Human-robot interactions: Creating synergistic cyber forces. pages $177-184$, 012002.

16. R Simpson, J Cullip, and J Revell. The cheddar gorge data set. BAE Systems (Operations) Limited, UK, Tech. Rep., 2011.

17. Mike Smith, Ian Baldwin, Winston Churchill, Rohan Paul, and Paul Newman. The new college vision and laser data set. The International Journal of Robotics Research, 28(5):595-599, 2009.

18. Melonee Wise, Michael Ferguson, Daniel King, Eric Diehr, and David Dymesich. Fetch \& freight : Standard platforms for service robot applications. 2016. 